\title{
Formarnos y autoformarnos en alfabetización informacional. Un programa de mentorización en bibliotecas universitarias-CRAI
}

\author{
Maria Pinto Molina * \\ Alejandro Uribe Tirado **
}

Artículo recibido:

6 de agosto de 2010.

Artículo aceptado:

25 de octubre de 2010.

\section{RESUMEN}

La Alfabetización Informacional (ALFIN) es un requisito de formación que desde los servicios académicos y especialmente de las bibliotecas-CRAI de las universidades, se debe ofertar a todos sus miembros en distintas modalidades y niveles. Para lograr buenos resultados es necesario que los responsables de los programas de ALFIN, además de tener buenos conocimientos sobre esta temática (modelos, normas, buenas prácticas), ofrezcan adecuadas habilidades-actitudes comunicativas, tecnológicas, documentales y didácticas. La "mentorización" (mentoring) es una de las estrategias organizacionales que dichas instancias universitarias pueden asumir considerando su fortaleza como medio

* Universidad de Granada, España. mpinto@ugr.es

** Universidad de Antioquia, Colombia. auribe@bibliotecologia.udea.edu.co

INVESTIGACIÓN BIBLIOTECOLÓGICA, Vol. 24, Núm. 52, septiembre/diciembre, 2010, México, ISSN: 0187-358X. pp. 63-95 
de formación y autoformación. El personal bibliotecario y el docente que aspire a ser formado (tutelado) por los formadores ya experimentados (mentores) y las personas a cargo de la formación organizacional (coordinadores) pueden crecer en dichas competencias, y así lograr mejores procesos y resultados de enseñanzaaprendizaje en los programas universitarios de ALFIN

Palabras clave: Alfabetización informacional; Mentorización; Formación; Autoformación; Profesionales de la información; Bibliotecarios; Bibliotecas universitarias-CRAI; universidades

\begin{abstract}
Training and self-training in information literacy: A program of mentoring in university libraries Maria Pinto Molina and Alejandro Uribe Tirado

Information Literacy (INFOLIT) in university academic services, libraries and learning resources centers is a training requirement that ought to be offered to personnel at diverse levels and through several approaches. In order to achieve excellent outcomes, the INFOLIT program leader must have good knowledge of models, standards and good practices in order to offer adequate skills and attitude sets in term of communication, technology, documentation and didactics. In light of its effectiveness in the realm of training and self-training, formal mentoring is a valuable organizational strategy university authorities can deploy. Librarians and academics wishing to be trained, as well as experienced trainers (mentors) and organizational training coordinators may grow into these competencies and thereby improve both process and outcomes of INFOLIT teaching-learning within the university.
\end{abstract}

Key words: Information literacy; Mentoring; Training; Self-training; Information professionals; Librarians; University libraries- Resource Centers for Learning and Research (RCLR); universities 


\section{El PROFESIONAL DE LA INFORMACIÓN EN EL CONTEXTO DE ALFIN}

U na biblioteca universitaria-CRAI ${ }^{1}$ plenamente integrada en el escenario académico y formativo, y que colabore con el equipo docente, constituye uno de los principales pilares para el aprendizaje global y autónomo del estudiante en los nuevos modelos de enseñanza (didácticas activas desde la perspectiva socio-constructivista), ya que le ofrece la adquisición de competencias para saber acceder a las diversas fuentes de información, saber analizarlas críticamente y transformar la información seleccionada en conocimiento. En este sentido la Alfabetización Informacional -ALFIN- tendría que abarcar de modo integral y holístico la enseñanza/aprendizaje de las competencias nucleares informacionales para saber acceder, buscar, seleccionar, valorar, reelaborar, comunicar y usar la información, con el fin de conseguir conocimientos y transmitirlos.

La ALFIN está siendo desde hace varios años objeto de atención tanto por parte del sector bibliotecario para defender la dimensión educativa de la biblioteca (ACRL/ALA, 2000, 2003; SCONUL, 2004, ANZIIL, 2004), como por parte del sector académico para contribuir a la formación de las competencias del estudiante (Pinto, Sales y Osorio, 2008).

La bibliografía sobre ALFIN es variada y está en crecimiento (Pinto, Cordón y Gómez, 2009). La educación de usuarios, propia del ámbito educativo, ha sido campo fértil para la evolución de algunos aspectos de la ALFIN, especialmente desde el ámbito de las bibliotecas, donde investigadores y profesionales se hallan inmersos en esta área de trabajo y estudio, contribuyendo a su avance. La certeza de que cada persona debe ser capaz de desarrollar sus potencialidades en un aprendizaje continuo a lo largo de toda la vida, hace que el tema requiera de una reflexión intensa y actualizada en la educación formal y por tanto, de su

1 "Hoy por hoy la universidad [...] está inmersa en una etapa reflexiva y dinámica y vive una revisión en lo que respecta a los contenidos, a las formas y a los medios destinados a enseñar y a aprender. El papel que desempeña en la actual sociedad de la información como productora, transmisora y difusora de conocimientos le otorga un protagonismo esencial en la formación y el desarrollo de los ciudadanos del siglo XXI, por lo que debe asumir una gran responsabilidad para afrontar los cambios[...] En este contexto, la biblioteca universitaria (BU) puede convertirse en fructífero eje de este proceso, en el marco de su transformación como Centro de Recursos para el Aprendizaje y la Investigación (CRAI). La creación de verdaderos [CRAI] puede plantearse como una apuesta emergente y estratégica de las universidades para concentrar y rentabilizar sus servicios de apoyo a la comunidad universitaria y para potenciar el trabajo en equipos que gestionen mejor la información y el conocimiento, desarrollando un proyecto global e integrador de los servicios que apoyan los procesos de enseñanza, aprendizaje e investigación. La presente obra pretende abordar esta potencialidad desde el paradigma educativo de la alfabetización informacional-ALfiN". (Pinto, M., Sales D. y Osorio, P., 2008) 
integración en el marco reflexivo y docente de una universidad alfabetizada en información (Webber, 2006; Webber y Johnston, 2006; Uribe Tirado, 2010a).

Es preciso señalar que para que haya una formación eficaz en ALFIN, los formadores, ya sean bibliotecarios o profesores, o ambos, tienen que perfeccionar sus propias destrezas en el uso, manejo y evaluación de la información. Por tanto el conocimiento de las normas (ACRL/ALA, SCONUL, ANZIL, DHIMéxico...) y los modelos didácticos de ALFIN (Big 6, Big Blue, Bruce, Kuhlthau...), se convierten en una gran e imperiosa necesidad para ser aplicados a los programas educativos, extendiendo el proceso formativo a bibliotecarios y educadores, con el fin de medir el nivel de competencia en el acceso y uso de la información y determinar las necesidades formativas para mejorar el nivel de aprendizaje y potenciar la eficacia de la institución. De esta forma la ALFIN se relaciona con los procesos evaluadores de modo que cada institución debe considerar en qué medida la ALFIN contribuye a mejorar los resultados de aprendizaje de sus estudiantes, y ayuda a producir graduados competentes y profesionales capacitados para el aprendizaje a lo largo de la vida.

En España, y como referente para Iberoamérica, destaca la apuesta de la Red de Bibliotecas Universitarias-REBIUN ${ }^{2}$ por la ALFIN a partir de su Plan Estratégico (2007) que se evidencia en algunos hitos como la elaboración de un documento sobre Competencias informáticas e informacionales en los estudios de Grado (2009) y la traducción/adaptación de la Guía de Buenas prácticas para el desarrollo de las competencias informacionales en las universidades españolas de CAUL (2008). También sobresale un estudio exploratorio que analiza la percepción que tiene de ALFIN un grupo de bibliotecarios españoles (Pinto, Sales, Osorio, 2009), al destacar que hay algunos que carecen de una visión estratégica de ALFIN como competencia nuclear en el currículo de los estudiantes. Además, adolecen de una falta de conocimiento sobre lo que éstos deben saber para poder manejar con solvencia las competencias informacionales nucleares, especialmente las relacionadas con la organización y utilización de la información, la creación de nuevo conocimiento y el uso ético y responsable de la misma. Generalmente, hay quienes han participado en actividades esporádicas y parciales de formación en ALFIN y no han colaborado en el diseño y desarrollo de un programa integral e integrado en la actividad curricular del estudiante, aunque con la implementación del Espacio Europeo de Educación y los nuevos títulos de grados poco a poco se están dando avances en esta línea. ${ }^{3}$ En este sentido, no sólo en España sino también en el contexto iberoamericano, son proporcionalmente mucho menos

2 Ver: http://www.rebiun.org/bibliotecas.html (72 bibliotecas universitarias) 
las bibliotecas universitarias que imparten formación reglada dentro de los programas académicos entre las aún pocas que desarrollan programas de ALFIN (Lau et al, 2007; Uribe Tirado, 2010b; Infolit Global/IFLA-UNESCO, 2010; Informe ALFIN-REBIUN 2010).

Los motivos por los que la mayoría de los bibliotecarios se consideran personas alfabetizadas en información tienen relación con su trabajo profesional, como responder a las necesidades y demandas de información de los usuarios; colaborar en la selección, acceso y valoración de la información; facilitar el apoyo a la docencia e investigación; enseñar los recursos de la biblioteca, y afrontar la formación de usuarios. Consideran que por el hecho de ser bibliotecarios son infoalfabetos, sin saber qué aportaciones les supone el paradigma emergente de ALFIN para su autoformación y la formación de los demás. Cuando se les interroga sobre las actividades de alfabetización realizadas en sus bibliotecas, la mayoría se refiere principalmente a la formación de usuarios en el manejo de fuentes de información y al conocimiento de los servicios de la biblioteca (Pinto, Sales, Osorio, 2009).

Paralelamente, si se analizan los distintos planes de estudio de biblioteconomía y documentación, se identifica que ha habido en buena medida presencia de cursos curriculares en formación de usuarios, habitualmente integrados con los de estudios de usuarios, pero desde una perspectiva tradicional de formar para usar los servicios de la biblioteca. No obstante, si se tiene en cuenta todo lo que implica ALFIN en conocimientos temáticos y en formación de competencias pedagógicas, sólo en los últimos años se han comenzado a incluir cursos desde esta perspectiva, aunque en contados programas, por lo que muchos de los nuevos profesionales están egresando sin haber tenido opciones de aprendizaje sobre todas las competencias que implica ALFIN para sí mismos y para ser formadores de usuarios del siglo XXI (Lau et al, 2007; Gómez y Licea, 2008; Pinto, M., Sales D. y Osorio, P., 2008, Uribe Tirado, 2006 y 2008; Licea, 2009; Guerra y Lahera, 2010).

Por consiguiente hoy en día tanto los profesionales de la información graduados, como los que están por graduarse, se encuentran ante el reto de aprender, comprender y enseñar las competencias informacionales, integrando los conocimientos alcanzados durante el desarrollo de su actividad con los nuevos paradigmas, modelos y métodos que requiere la formación basada en competencias. El bibliotecario tendrá que estar alfabetizado en información para resolver problemas complejos relacionados con la gestión de la información y poder a su vez formar a otros profesionales y usuarios de la comunidad universitaria.

En esta línea se pronuncia Wasik (2007), quien afirma que los profesionales de la información deben tener solvencia en estas competencias: dominio 
de las tecnologías, habilidades para los procesos de referencia virtual y el uso de recursos digitales, habilidades para la evaluación del servicio, habilidades para la creación de procedimientos innovadores, babilidades como instructor, habilidades para la colaboración y la participación en la comunidad virtual, habilidades en el uso de bases de conocimiento.

Es decir, debe ser un profesional integral bien formado y que lleve a la práctica sus competencias del saber, del hacer, del ser, y del aprender a convivir y trabajar juntos (UNESCO, 1996).

Es esta coyuntura de cambio en la que emerge con fuerza el paradigma de la ALFIN como faro para la sociedad de la información y el conocimiento (Declaratoria ALFIN Alejandría / Garner, 2006), por lo que el profesional de la información con experiencia debería desempeñar la labor de tutor y guía en los procesos de formación de los recursos humanos, del capital humano y de la institución, generando un proceso de aprendizaje y docencia en cascada que lo transforme en co-formador de otros colectivos. Pero a su vez, teniendo en cuenta la máxima "de que para formar hay que estar formado", él mismo debería estar formado e integrar su competencia informacional con el resto de las competencias propias de la profesión.

En este sentido es necesario desarrollar acciones de mejora formativas y fomentar alianzas claras, desde la mutua confianza, entre la institución docente universitaria y la biblioteca-CRAI. El camino hacia el CRAI de alguna manera ha activado la implicación del personal bibliotecario en las tareas docentes e investigadoras universitarias, aunque hace falta una mayor organización (y formación interna) a este respecto. La participación de los bibliotecarios en el proceso formativo de ALFIN es un tema emergente de estudio, no solo por las actividades formativas realizadas, sino por la necesidad de contar con un adecuado entrenamiento en cuanto a la competencia informacional y al uso de renovadas estrategias pedagógicas. En esta línea se pronuncian Sowell (2003), Donnelly (2003) y Portmann y Julius (2004), quienes afirman que lo significativo sería definir, por parte del bibliotecario, qué estrategia pedagógica es más efectiva para aplicar en el proceso de enseñanza de ALFIN.

\section{QUÉ ES MENTORIZACIÓN Y SUS POTENCIALIDADES DE FORMACIÓN Y AUTOFORMACIÓN}

El término mentorización o mentoring -considerando esta expresión como la traducción más común pero aún sin acuerdos al respecto, y siendo el anglicismo la expresión más presente incluso en la literatura académica en español- es utilizado para sugerir un proceso de expansión y profundización de buenas prácticas 
y hábitos hacia otros, tanto en el ámbito académico y profesional como en otros entornos de la vida (Mullen y Lick, 1999, Zachary, 2000, 2005). Parafraseando a Lary (1998), la mentorización es la relación de apoyo profesional entre un experimentado y exitoso empleado que está a mitad de su vida laboral y un empleado que apenas inicia. Este es un método que implica alentar a los nuevos talentos a compartir conocimientos y conexiones, que les facilite de forma más rápida su crecimiento profesional y laboral. Es decir, la mentorización es un recurso de cambio y de mejora para la organización que lo aplica.

Este concepto ha sido definido en la literatura de múltiples formas y hasta interrelacionándolo con otros conceptos-prácticas en diferentes áreas del conocimiento y sectores de la sociedad. ${ }^{4}$

En este sentido Penny (2008) afirma que es necesario indicar que aunque se usan de forma indiscriminada los términos coaching, counselling y mentoring, no tienen el mismo significado ni connotaciones, pues

cada método tiene un enfoque decididamente diferente. Coaching se centra en lo que está sucediendo -el aquí y ahora-... El counselling se centra en los comportamientos del pasado... mientras que las relaciones de mentoring se focalizan en el futuro, para desarrollar grandes potenciales.

Por tanto, y teniendo en cuenta diferentes definiciones y perspectivas de mentorización como bien recopila Soler (2003): “considerando la visión norteamericana (Allen; Bell; Biehl; Hendricks; Jonson; Murray; Peddy; Shea; Wickman y Sjodin; Lary; Luecke, entre otros) y la visión europea (Andey, Early y Foster; Clutterbuck y Megginson; Coway; Hay; McLenann; Mumford; Parsloe; Clarke; Zey, entre otros)", desde este artículo resaltamos y acogemos como guía para nuestra propuesta, las definiciones que presentan Shea (1994) y Andey, Early y Foster (1999), respectivamente, pues exhiben un panorama integral de esta estrategia, potenciar para... enseñar $y$ aprender, como indica el proverbio chino, como busca la ALFIN:

La mentorización consiste en desarrollar, cuidar, compartir y ayudar a mantener en una relación en que una persona invierte su tiempo, su saber-como (knowhow) y su esfuerzo para potenciar el desarrollo de otra persona, en el ámbito de los conocimientos y las habilidades, y así dar respuestas a necesidades críticas en la vida de esa persona en direcciones que le preparen para una productividad mayor o un éxito futuro... La mentorización es ayudar a los empleados (a otros) a descubrir todo su potencial. 
La mentorización se presenta cuando una persona ayuda a otra en su desarrollo, a adquirir nuevos conocimientos y habilidades, a interiorizar puntos de vista y desarrollar todo su potencial.

La mentorización presenta diferentes categorías, determinadas por el grado de estructuración y formalidad de las mismas en su realización práctica, en el tipo de interrelación entre mentor y tutelado, y entre ambos y el lugar que se le da o no a esta relación de aprendizaje mutuo dentro de las políticas de formación-capacitación en la organización a la que pertenecen.

Retomando a Shea (1997, 1999, 2002, 2003), la estrategia de mentorización se divide en varios tipos de intercambio de acuerdo con el grado de formalidad-estructuración y con la duración de la relación entre el mentor y el tutelado:

- La mentorización poco estructurada, de intercambios momentáneos (Informal)

Se produce cuando en las relaciones diarias entre los empleados, los más conocedores de un tema, de una actividad, ayudan a otros poco conocedores, pero no implica una acción directa promovida por la organización, sino que está centrada en la buena actitud y disponibilidad de los empleados, quienes asumen un determinado rol, que es facilitado o no indirectamente por la cultura y clima organizacional que facilita el trabajo colaborativo.

- La mentorización más estructurada, a corto plazo (Situacional)

La relación se genera sólo en un momento dado, con una directriz organizacional y durante un período de tiempo muy corto para resolver un problema concreto, pero resuelto dicho problema o pasado ese momento específico, la relación de enseñanza-aprendizaje termina entre quien tuvo el rol de mentor y de tutelado.

- La mentorización estructurada, a medio y largo plazo, según los requerimientos (Formal)

Es un programa que se desarrolla como política de la organización y se supone como consciente de los beneficios productivos que conlleva el aprendizaje y la gestión de conocimiento, en el que son formados tanto el mentor como el tutelado, tras procesos previos de motivación, divulgación y selección, para así poder desempeñar de forma competente sus roles. Como programa tiene una estructura concreta, planificada y que realiza una evaluación continua; a su vez, tiene un tiempo de inicio y de finalización según los objetivos esperados a mediano (meses) y largo plazo (años). 
En este artículo apostamos por la mentorización formal como estrategia fundamental para las bibliotecas universitarias-CRAI dada la necesidad de que sus profesionales se formen y sean formadores en ALFIN, pero no descartamos la importancia complementaria del aprendizaje organizacional que conlleva la mentorización informal o situacional. Desde esta perspectiva formal de su aplicación se hace necesario tener en cuenta que un programa de mentorización, como indican Bey y Holmes (1992), presenta algunas características claves:

- es un proceso y una función compleja que requiere una estructura adecuada y de una gran sensibilidad para acomodarse a las diferentes situaciones,

- implica apoyo, ayuda y orientación pero no una evaluación de la persona tutelada y

- requiere tiempo para que mentores y tutelados entren en contacto y se comuniquen.

Además este programa debería adaptar los siguientes siete postulados indicados por Mullen y Lick (1999):

- La mentorización es posible para todos.

- La mentorización es una búsqueda de aprendizaje permanente.

- La mentorización no es sólo una relación profesor-alumno en el sentido convencional, sino que implica una amplia gama de capacidades que deben desarrollarse de manera adecuada entre el mentor y el tutelado.

- Diferentes personas tienen diferentes necesidades de orientación con base en sus habilidades para interrelacionarse. Las necesidades de orientación de cada uno son moldeadas por situaciones particulares de su vida (por ejemplo, la jubilación, la escolaridad, los primeros momentos de la práctica profesional).

- Diferentes personas tienen diferentes necesidades, y también distintas habilidades y conocimientos que determinan la necesidad de orientación, la forma y el trabajo colaborativo que implica la mentorización. (uno no puede aplicarles las mismas estrategias a toda persona, ni puede esperar las mismas habilidades y conocimientos de un tutelado).

- La mentorización no implica un orden jerárquico rígido, de status, de enseñanza-aprendizaje en un único sentido. Más bien el potencial de la mentorización es un proceso mutuo de enseñanza-aprendizaje entre 
mentor y tutelado. No obstante algunas veces es necesario considerar que ante una situación dada es necesario un proceso más tradicional de enseñanza-aprendizaje, que seguir una estrategia de mentorización, lo cual implica analizar cada situación, cada organización: "cada organización tiene su propia necesidad y forma de mentorizarse”.

- El potencial de los mentores para aprender, y de los tutelados para enseñar, reevalúa los procesos tradicionales de generación y utilización del conocimiento. En la mentorización los roles de enseñanzaaprendizaje se intercambian y se hacen en la práctica a veces imperceptibles, aunque cada uno tiene un rol desde el punto de vista de un programa formal.

Todo esto implica que el proceso de mentorización debe ser protagonizado por profesionales expertos y experimentados, preocupados por mejorar su actividad profesional y ser capaces de reorientar las necesidades del profesional principiante (junior) en distintos frentes, como el psicológico, cognitivo, comunicacional, estratégico, etcétera.

También el mentor debe transmitir la cultura de la organización a la que pertenece y ha de poseer las siguientes características (Flores, 2006):

- personales: empatía, flexibilidad, afán de apoyo, facilidad para la comunicación, diplomacia,

- profesionales: capacidad para transmitir la cultura de la organización a la que pertenece; habilidad en la gestión de las clases; habilidad para transferir los conocimientos; capacidad de cambio; capacidad para planificar y organizar; capacidad de compartir conocimientos y experiencias (Vanzant, 1980, Cronan et al., 1986).

- sociales: habilidad para trabajar con otros; habilidades comunicativas; capacidad para guiar; tenerse confianza y respeto, y ser responsable.

El mentor ha de ser formador de formadores y ha de contribuir a mejorar la calidad formativa de la persona tutelada. Por lo que también necesita recibir formación, orientación y apoyo de la comunidad universitaria (Moir, 2003).

Finalmente acercando todo lo dicho a la práctica en las bibliotecas universitarias-CRAI, para iniciar la planificación y el desarrollo de procesos de mentorización formal es necesario contar con la presencia de:

- Bibliotecarios seniors o expertos, que tengan más de cinco años de experiencia profesional, un alto nivel de conocimiento y destrezas, y que 
estén preparados para un aprendizaje eficiente a lo largo de la vida y sean capaces de combinar competencias y capacidad de innovación.

- Bibliotecarios juniors o principiantes, que además de su competencia profesional posean un conjunto de habilidades cognitivas encaminadas a cultivar la capacidad de reflexionar, de evaluar y aprender de su entorno, y en particular de los bibliotecarios expertos.

A su vez habrán de promoverse desde el inicio del proceso de planificación y desarrollo, y de manera continua, formas de comunicación proactiva para la implementación de estrategias diversas de formación que incidan en los resultados del aprendizaje, tales como:

- La celebración de seminarios que tengan en cuenta las expectativas sociales y profesionales de los profesionales de la información del siglo XXI.

- El uso de los ciclos de mejora como estrategia para ayudar el proceso reflexivo de aprendizaje, tanto en mentores como en mentorizados.

- Talleres de análisis que incluyan procesos de reflexión compartida.

- Grupos de discusión que tengan como epicentro el tema de aprender.

- Una pedagogía basada en las TIC que promueva la construcción y uso del portafolio electrónico, el empleo del chat y el correo electrónico en el intercambio de experiencias.

- Enseñar fomentando la adquisición de habilidades cognitivas complejas, utilizando recursos varios como, mapas, juegos, videos, etcétera.

\section{EXPERIENCIAS DE MENTORIZACIÓN EN BIBLIOTECAS}

Si se hace una revisión de la literatura especializada en relación con la ciencia de la información, la bibliotecología y la documentación, observamos que aunque lleva ya varios años en desarrollo en diferentes organizaciones, el tema de mentorización -sobre todo en una perspectiva formal -no ha estado muy presente en las bibliotecas las últimas dos décadas, aunque sí se haya dado en la perspectiva informal o situacional (Bonnette, 2004; Farmer, Stockham y Trussell, 2009).

Las pocas bibliotecas que han efectuado prácticas o programas formales de mentorización se han concentrado preferentemente en las bibliotecas académicas-universitarias y algunas asociaciones de los Estados Unidos -reseñadas en las referencias de este artículo-, aunque también se han reportado algunas experiencias desde finales de los noventa hasta hoy en Suecia (Karolinska 
Institutet University Library), ${ }^{5}$ Inglaterra (British Library Research and Innovation Centre), ${ }^{6}$ Australia (Australian Library and Information AssociationALIA $/,{ }^{7}$ Canadá (Association of Canadian Map Libraries and Archives) ${ }^{8}$ Hong Kong (Institute of Education Library), ${ }^{9}$ Suráfrica (National Library of South Africa) ${ }^{10}$ entre otras.

El hecho de centrarse en bibliotecas académicas-universitarias se debe a que es este tipo de biblioteca el que lidera las innovaciones organizacionales en este sector (bien sea por su mismo contexto muy relacionado con la innovación, la generación y la aplicación de nuevas prácticas organizacionales, o por contar con mayores posibilidades en cuanto a capital bumano y recursos económicos y tecnológicos); no obstante, también encontramos algunos casos de mentorización en algunas bibliotecas públicas de los Estados Unidos (Shaping Public Library, ${ }^{11}$ New Jersey's Libraries) ${ }^{12}$ o en Canadá (Mississauga Library System's). ${ }^{13}$

En los casos particulares de programas de mentorización desarrollados por las bibliotecas académicas-universitarias, la mayor motivación para su formulación e implementación, ha sido la preocupación existente de que antes del 2010, un $40 \%$ del personal que ha liderado las bibliotecas las últimas décadas se jubilará, y entre el 2010 y el 2020 esta tasa de jubilación se incrementará en un 27\% (Munde, 2000), lo cual que implica que cerca del $70 \%$ del personal de las bibliotecas en el inicio de la próxima década será junior o principiante.

Por tanto para la dirección de esas bibliotecas y las universidades que las acogen fue evidente la gravedad e importancia de esa situación, y se comenzó entonces, como parte de planes de relevo generacional, el desarrollo de los programas de mentorización (de captura y transferencia de conocimientos) para que toda la experiencia de los bibliotecarios seniors o expertos no terminara en sus casas, sino en la biblioteca, en los bibliotecarios juniors, y que complementara y potenciara los nuevos conocimientos y estrategias que éstos podían aportar para modernizar y prestar un mejor servicio acorde con las exigencias tecnológicas en cuanto a contenidos y aprendizaje, de la actual sociedad de la información, del conocimiento y del aprendizaje.

Ver: (Haglund, 2004)

Ver: (Nankivell, Clare y Michael Shoolbred, 1997)

Ver: (Ben, J; Brennand M., 2008)

Ver: (ASCMLA, 2002)

Ver: (Sit,2003)

10 Ver: (Golian, L.M. y Galbraith, M.W., 2006)

11 Ver: (Gibson, R., 2003)

12 Ver: (NJLA, 2009)

13 Ver: (Peer Systems Consulting Group Inc, 2009) 
Entre esas bibliotecas académicas-universitarias se han destacado, considerando las referencias constantes en la literatura especializada, ${ }^{14}$ las siguientes experiencias en algunas universidades norteamericanas:

- Albany University

- Arizona State University

- Cornell University

- Kansas State University

- Louisiana State University

- Middle Tennessee State University

- Northwestern State University

- Rutgers University

- State University of New York

- University of California-UCLA

- University of Cincinnati

- University of Connecticut (Acad. Medical Library)

- University of Delaware

- University of Georgia

- University of Maryland (Geog. Inf. Systems-GIS)

- University of Southern California

- University of Utah

- University of Virginia

- Washington University

- Yale University

A su vez, complementando a la labor particular de dichas bibliotecas, varias asociaciones profesionales ${ }^{15}$ también han liderado experiencias en distintas bibliotecas universitarias, y en menor medida, en bibliotecas públicas y escolares:

- Chinese American Librarians Association

- Florida Library Association

- Illinois Library Association

14 Ver: (Wittkopf B., 1999 / Culpepper, J. C., 2000 / Bullinngton, J.S. y Boylston, S.D., 2001 / University of Southern California Libraries, 2001 / Wang, H., 2001 / Golian, L.M., 2003 / University of Utah., 2003 / Cornell University Library, 2004 / Martorana, J. et al., 2004 / Demirhan, A., 2005 / Mosley, P. A, 2005 / Meloni, C., 2006 / University of Maryland Libraries, 2006 / University of Georgia Libraries, 2006 / Hilbun, J. y Akin, L., 2007 / Washington University Libraries, 2007 / Yale University Library, 2007 / Arizona State University Libraries, 2009/Library Leadership Network, 2009)

15 Ver: (Nankivell, C. y Shoolbred, M., 1997 / Special Libraries Association, 2001 / Studwell, W.E., 2002 / Haglund, L., 2004 /Davidson, J.R., y Middleton, M.A., 2006 / Farmer, D.; Stockham, M. y Trussell, A., 2009 / Florida Library Association, 2010) 
- Medical Library Association

- Montana Library Association

- New York Library Association

- Southeastern Library Association

- Wisconsin Library Assocation

Finalmente han sido igualmente relevantes los macroprogramas llevados a cabo por las siguientes asociaciones de mayor alcance en Estados Unidos, y con gran influencia en otros países:

- American Library Association (ALA) ${ }^{16}$

- Association of Research Libraries (ARL) $)^{17}$

- Association of College and Research Libraries (ACRL) ${ }^{18}$

- Society for American Archivists (SAA) ${ }^{19}$

- Special Libraries Association (SLA) ${ }^{20}$

- The National Association to Promote Library \& Information Services to Latinos and the Spanish Speaking (REFORMA) ${ }^{21}$

Entre estas múltiples experiencias, artículos de tipo divulgativo/descriptivo que presentan por qué iniciaron un proyecto de mentorización, su estructura y algunas veces sus resultados considerando la opinión de los participantes, destacamos la siguiente síntesis sobre el desarrollo de proyectos de mentorización en dichas bibliotecas universitarias y asociaciones, y las siguientes afirmaciones:

Los valores organizacionales son aprendidos tanto a través de la observación de los miembros de la organización, como a través de las declaraciones de misión, objetivos, políticas y procedimientos. Los colegas son modelos importantes que pueden interpretar los valores de la organización y ayudar a reducir los sentimientos de aislamiento. El nuevo bibliotecario puede mejorar su integración con la cultura organizacional, utilizando el conocimiento del mentor, el desarrollo de aliados útiles, y mostrando una voluntad de llevar a cabo los objetivos de la biblioteca, y el pensar con creatividad (William K. Black y Joan M. Leysen, University Library, Middle Tennessee State University).

$16 \mathrm{http} / /$ www.ala.org/ala/mgrps/rts/nmrt/oversightgroups/comm/mentor/mentoringcommittee.cfm

17 http://www.arl.org/diversity/init/

18 http://www.ala.org/ala/mgrps/divs/acrl/about/sections/is/projpubs/mentoring/index.cfm

$19 \mathrm{http}: / / \mathrm{www} 2$.archivists.org/groups/mentoring-program-subcommittee 
El mentoring es una estrategia probada para comprometer, desarrollar y retener a los líderes de la próxima generación. También es una excelente metodología para la transferencia de los conocimientos prácticos y, más importante, [...] la sabiduría y experiencia (por lo general de los más mayores) a los más jóvenes... El mentoring tiene la capacidad de cambiar a la gente. Por su carácter estratégico para su futuro, para tomar las riendas de su carrera y la responsabilidad de su propio desarrollo profesional, cualquier cosa puede suceder...

(Jill Benn y Michelle Brennand, Australian Library and Information AssociationALIA).

\section{Claves para estructurar un Programa de mentorización en Alfin PARA BIBLIOTECAS UNIVERSITARIAS}

La propuesta genérica de mentorización que ofrecemos se articula en un modelo cíclico sistémico-situacional, centrado en la formación-autoformación ALFIN y basado en fases, estrategias y recursos (Kuyper-Rushing, 2001).

Retomando las diferentes propuestas llevadas a cabo en las bibliotecas universitarias y las diferentes características y postulados de un programa de mentorización mencionadas anteriormente, y acogiendo el modelo de "Lifelong mentoring” (Mullen y Lick, 1999), podemos inferir que un programa de mentorización formal debe considerar la integración transversal de tres componentes: necesidades, habilidades y recursos, y asumir las siguientes premisas: un programa formal de mentorización no se impone, se construye...; el éxito o no de un programa formal de mentorización estará dado si hay un buen proceso de motivación y divulgación de sus beneficios...; ser mentor o tutelado no se asigna como obligación, es resultado de un gusto mutuo por aprender y enseñar para beneficio personal y organizacional. ${ }^{22}$

Teniendo siempre presente lo anterior, el programa de mentorización formal para ALFIN que proponemos se articula en estas ocho fases, incluyendo algunas estrategias generales y pautas en los recursos, pues cada contexto es distinto e implica por tanto adecuarse a sus particularidades: 


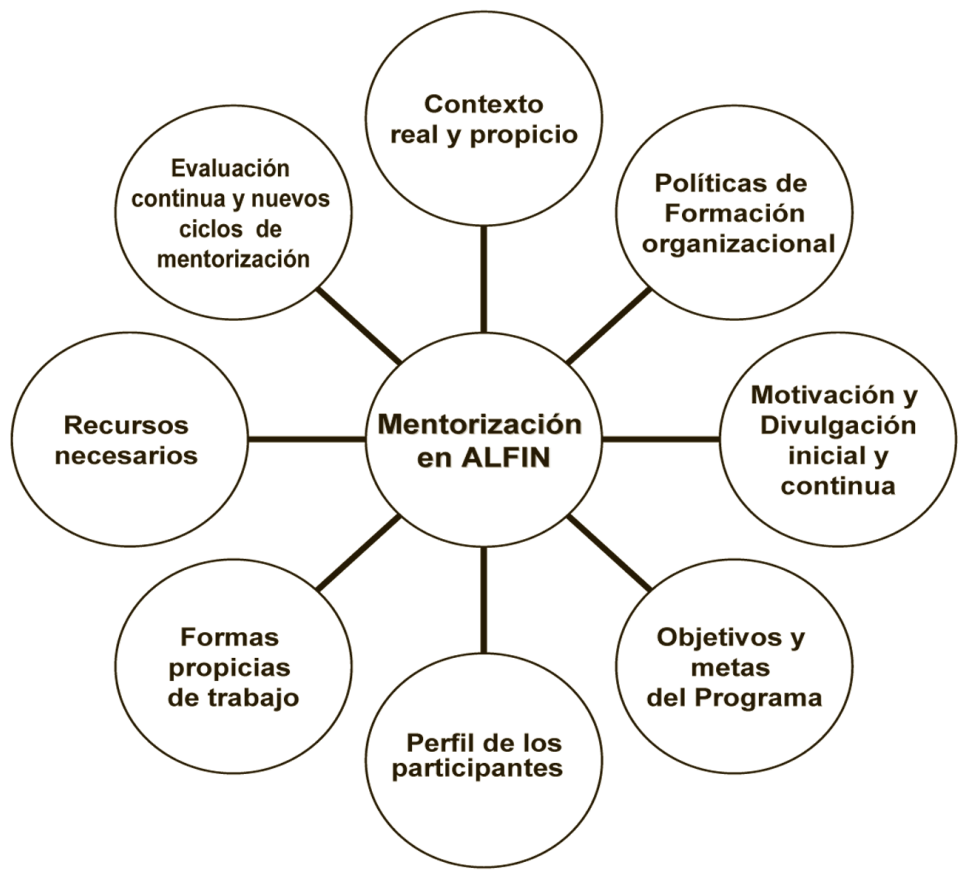

a. Contexto real y propicio: social, laboral, educativo, organizacional, personal Este primer aspecto implica la realización de un diagnóstico organizacional desde el área-proceso que se encarga habitualmente de la formación de usuarios, o en términos más actuales, de la formación en competencias informacionales teniendo en cuenta los siguientes aspectos a identificar:

- La historia y realidad actual en la biblioteca respecto a los programas de formación de usuarios (visión tradicional en formación en los servicios de la biblioteca o de ALFIN como un componente más), o presencia de una visión más actual e integral que conciba la ALFIN como parte central del desarrollo de toda biblioteca universitaria-CRAI. Esta historia permite identificar en qué se ha avanzado, con qué recursos se ha contado, qué momentos específicos y personas han liderado esa área-proceso en un tiempo determinado (sus visiones favorables o no hacia la perspectiva de ALFIN) y cuáles han sido las mejores prácticas y lecciones aprendidas (tanto lo bueno como lo malo) que esto ha dejado.

- Realizar un inventario de los conocimientos, habilidades y actitudes (competencias) que el personal de la biblioteca tiene, sus 
perfiles de formación e historia de desempeño profesional en esta biblioteca y antes de trabajar en ella, para así tener los insumos para elaborar una base de datos de lo que comúnmente en gestión del conocimiento se denomina "páginas amarillas", para con ello reconocer necesidades de formación y habilidades (expertos $y$ junior en ALFIN). En este caso específico se haría énfasis en identificar las competencias presentes y esperadas de un profesional alfabetizado informacionalmente conforme a los estándares internacionales o nacionales-regionales-organizacionales (si los hubiese) de Alfabetización Informacional a nivel universitario.

- Investigar si en la universidad, y en la biblioteca ha habido anteriores intentos para desarrollar un programa de mentorización, bien fuera de forma general o para determinadas áreas, y así identificar si es un tema conocido, si se planteó y no se desarrolló y por qué se dio esa situación, o si se está realizando ya un programa de este tipo y no es muy conocido en toda la universidad o la biblioteca misma, por falta de divulgación. Complementariamente, habrá que identificar si se han desarrollado programas orientados a buscar ser "una organización que aprende" o que "gestiona el conocimiento", pues entre estos programas y la mentorización hay una fuerte interrelación.

- Conocer qué bibliotecarios y profesores-investigadores están, tanto en la práctica como en la teoría, interesados en la ALFIN y la visualizan como el desarrollo de competencias para el aprendizaje permanente.

b. Politicas de formación organizacional

Un programa de mentorización es un programa de formación (autoformación) ${ }^{23}$ organizacional, por lo que otra de las primeras acciones a realizar, además de las del punto anterior, y que permite una adecuada contextualización sería:

- Conocer/re-conocer si la universidad como un todo, o la biblioteca, tiene una política definida de formación que refleje su operatividad en un programa de formación a corto, mediano y largo plazo. Esto permitiría analizar si la formación es una acción continua y estructural (responde a una verdadera política) o una

23 Implica la autoformación, ya que aunque el mentor acompañe al tutelado en muchos procesos de su aprendizaje, debe vivir una actitud continua de ser más competente y buscar en todo momento estar formándose, sea acompañado o solo (leer, observar, analizar, preguntar); a su vez, en el caso del mentor, se da esta misma situación, pues en este proceso no siempre lo estará acompañando el coordinador y no todas las interacciones que le permitan aprendizajes en su rol de formador, se darán en compañía del tutelado. 
acción puntal, más orientada a "la capacitación por la capacitación” o la capacitación para hacer frente en forma más reactiva que proactiva a requerimientos para solucionar problemas urgentes o hacer frente a nuevas realidades no esperadas o como "modas".

- Identificar si como parte de esa política o programa, hay un inventario de las necesidades de formación del personal, y específicamente, cuáles se dirigirían, para el caso del personal interesado en ALFIN, al desarrollo de competencias informacionales, comunicativas, pedagógicas, tecnológicas...

- Saber si la universidad o la biblioteca gestionan un programa de incentivos internos (en dinero, en tiempo, en estudio, etc.) por formación o participación en proyectos determinados sin ser parte obligatoria de su desempeño.

Adicionalmente a estas acciones concretas, estaría una acción estratégica fundamental por su peso político-administrativo, por su trascendencia en la aprobación y apoyo institucional para la creación e implementación de un programa de mentorización, como es el vincular éste al desarrollo del Plan Estratégico de la Universidad y de la Biblioteca, para tener así un suficiente sustento organizacional del por qué hay que llevarlo a cabo. Esto desde la perspectiva de la necesidad constante de actualizar el personal para ser "una organización moderna", "una organización que aprende", "que gestiona el conocimiento"; y que reconoce que la Alfabetización Informacional y las competencias informacionales (como lo reconoce la UNESCO y otras organizaciones) son claves y necesarias para el ciudadano y para el universitario del siglo XXI.

c. Motivación y divulgación inicial y continua

Uno de los puntos que los diferentes ejemplos y modelos para llevar a cabo un programa de mentorización presentan en común es la realización de un plan de motivación continuo para dar a conocer en qué consistiría este programa y cómo vincularse a él. Y ya puesto en desarrollo el programa, para presentar los beneficios que se pueden obtener y testimonios positivos en ese sentido, y vincular a nuevos participantes (mentores-tutelados). En la parte inicial del proceso suele haber desconocimiento sobre aquello en lo que consiste un programa de mentorización, y sobre todo, muchos "temores-prevenciones" por un lado a lo "nuevo", y por otro, al aumento (obligatorio) de cargas laborales. Eso implica desarrollar acciones encaminadas a:

- Presentar en forma sencilla y sintética en qué consiste un programa de mentorización, su forma de funcionar y los beneficios 
tangibles o intangibles que produciría. Además de informar si en la institución hay un programa de incentivos y si la participación en el programa de mentorización conlleva a un determinado reconocimiento tanto a quienes tuvieran el rol de mentor como de tutelado.

- Recordar las tres premisas básicas: se construye, beneficia a cada uno de los que participen y a la organización, y no se obliga a participar.

A su vez, ya estando en desarrollo el programa de mentorización, se deben siempre retomar acciones de Motivación y Divulgación, que permitan periódicamente vincular al programa a nuevos y antiguos empleados-docentes (potenciales mentores o tutelados) quienes por diferentes circunstancias (vinculación reciente, falta de tiempo, desconocimiento, escepticismo) aún no lo conocían o no se habían vinculado al mismo. Esas acciones se encaminarían a presentar por diferentes medios y formatos, los resultados positivos del programa y los mejoramientos que se han realizado para que éste funcione mejor. En este punto los testimonios positivos son claves, y el tener realmente en cuenta las críticas de quienes ya participan en él, y a partir de ellas mostrar las acciones de mejora a corto, mediano o largo plazo que se han realizado, para que así se perciba que todos pueden potencialmente participar, los beneficios que se producen, la atención que se da a sus recomendaciones, etcétera.

d. Objetivos y metas del Programa

Como todo programa deberá tener objetivos y metas concretas que permitan medir el logro de sus propósitos. En este aspecto será necesario, según los resultados de los diagnósticos y análisis de los anteriores puntos 1 y 2, identificar el alcance que se le dará al programa de mentorización (entendiendo por alcance el número de participantes que habría y los recursos disponibles para ejecutarlo en un período establecido de 6 meses, uno, dos o tres años), o si por el momento no hay las condiciones para desarrollarlo debido a los resultados del punto 3: no hay buena aceptación, no se comprende el para qué realizarlo, no hay interesados o capacitados en participar según los distintos roles. Es decir, en la medida que haya un contexto más o menos propicio y un número determinado de interesados en participar, los objetivos y metas pueden ser mayores o no. Lo importante es definir ese alcance y según el mismo ser realistas en su formulación.

Definidos esos objetivos y metas, la preparación de los potenciales participantes del programa es la fase clave que nos ayudaría conocer lo que se espera de cada rol. 
e. Perfil de los participantes

En un proceso de mentorización son tres los agentes principales que participan cuando la dirección ya ha otorgado su visto bueno para seguir y se cumplen parcial o totalmente los puntos anteriores. Esos tres agentes, que son los que realmente viven la mentorización son el mentor, el tutelado y el coordinador (aunque éste es un rol más administrativo y designado por la dirección).

Como señalamos anteriormente y presentamos algunas características en epígrafes anteriores, cada participante tiene un rol específico, concretado en acciones evaluables de las que se aprenderá, pues nunca se puede olvidar que ésa es la riqueza de participar en un programa de mentorización: APRENDER... APRENDERJUNTOS... ENSEÑAR... ENSEÑARJUNTOS.

Cada uno de estos roles tiene ciertas características, y por tanto, criterios que deben ser seleccionados para un determinado rol, pues no todos los bibliotecarios, aunque estén interesados, podrán ser mentores o tutelados según el rol que estimen para sí, pues en algunos casos surge la arrogancia o la subvaloración, es decir, nos creemos mentores cuando nuestro nivel es de tutelados, o nos creemos tutelados cuando podemos ser mentores.

Éstas serían en forma general algunas de las características, actuaciones y beneficios que cada uno de los roles que participan en un programa de mentorización podría esperar, retomando a diferentes autores recopilados por Cahill y Blanchard (2001) y Soler Angels (2003). 


\begin{tabular}{|c|c|}
\hline \multicolumn{2}{|c|}{ Mentor } \\
\hline $\begin{array}{l}\text { Características } \\
\text { - Deseoso de ayudar } \\
\text { - Haber tenido una experiencia positiva en su forma- } \\
\text { ción cuando joven } \\
\text { - Tener una buena credibilidad y reputación } \\
\text { - Disponer de tiempo y deseos de enseñar-aprender } \\
\text { - Estar al día respecto a los conocimientos de su } \\
\text { área } \\
\text { - Responsable y comprometido } \\
\text { - Saber compartir } \\
\text { - Interés en apoyar el desarrollo de otros } \\
\text { - Poseer sentido del humor y "buen genio" } \\
\text { - Tener buenas habilidades interpersonales } \\
\text { - Mostrar confianza y seguridad cuando hay que ha- } \\
\text { cerlo } \\
\text { - Humilde } \\
\text { - Ser honesto con los demás }\end{array}$ & \begin{tabular}{l}
\multicolumn{1}{c}{ Actuaciones esperadas } \\
- Comunicarse con propiedad \\
- Proporcionar retroalimentación constante \\
- Guiar oportunamente \\
- Proporcionar en forma pertinente información \\
- Generar ideas \\
- Confrontar \\
- Alentar \\
- Explorar opciones
\end{tabular} \\
\hline $\begin{array}{l}\text { Criterios de selección } \\
\text { El mentor se puede seleccionar generalmente de tres } \\
\text { maneras posibles: se presenta voluntariamente, es } \\
\text { nombrado por el responsable del programa (el coor- } \\
\text { dinador) o por los propios tutelados que participan } \\
\text { en el programa. No obstante, para esta selección es } \\
\text { necesario identificar quiénes cumplen esas caracte- } \\
\text { rísticas para ser mentores con esos roles esperados, } \\
\text { para lo cual se pueden realizar: tests (especialmente } \\
\text { en el caso voluntario); entrevistas (cuando el coordi- } \\
\text { nador designa una persona que por su trayectoria en } \\
\text { el área, en el caso de ALFIN, no se debe descartar el } \\
\text { considerar algunos ítems del test: lo pedagógico); o } \\
\text { sesiones de brainstorming-lluvia de ideas (cuando lo } \\
\text { eligen los tutelados tras identificar entre los posibles } \\
\text { candidatos el mejor mentor, o entre ellos mismos los } \\
\text { más idóneos, para asumir ese rol). }\end{array}$ & $\begin{array}{l}\text { Beneficios: } \\
\text { - Desarrollar la intuición } \\
\text { - Desarrollar la paciencia y la tolerancia } \\
\text { - Incrementar su prestigio dentro de la organización } \\
\text { y externamente } \\
\text { - Adoptar ideas nuevas y frescas del tutelado } \\
\text { - Aprender a dar y recibir feedback } \\
\text { - Asumir nuevas responsabilidades } \\
\text { - Mejorar sus habilidades interpersonales } \\
\text { - Ser proactivo y constructivo para los otros y la or- } \\
\text { ganización } \\
\text { - Obtener satisfacción por el servir al desarrollo de } \\
\text { otros } \\
\text { - Obtener incentivos (si la organización los ofrece co- } \\
\text { mo política) }\end{array}$ \\
\hline
\end{tabular}




\begin{tabular}{|c|c|}
\hline \multicolumn{2}{|c|}{ Tutelado } \\
\hline \begin{tabular}{l}
\multicolumn{1}{c}{ Características } \\
- Ansioso por aprender y responsable de su propio \\
aprendizaje \\
- Curioso \\
- Con sentido del humor y "buen genio" \\
- Seguro de sí mismo \\
- Humilde \\
- Autocrítico y receptivo a las críticas \\
- Flexible \\
- Dispuesto a pedir ayuda y dejarse guiar \\
- Centrado: que tiene claro lo que quiere y espera \\
- Con grandes habilidades interpersonales
\end{tabular} & $\begin{array}{l}\quad \text { Actuaciones esperadas } \\
\text { - Ser proactivo } \\
\text { - Ser capaz de comunicarse } \\
\text { - Innovador } \\
\text { - Comprometido con la organización } \\
\text { - Constructivo en sus interrelaciones con los otros }\end{array}$ \\
\hline $\begin{array}{l}\text { Criterios de selección } \\
\text { Los tests son la principal herramienta utilizada pa- } \\
\text { ra identificar si el candidato a tutelado cumple esas } \\
\text { características y puede desempeñar plenamente } \\
\text { ese rol. Dichos tests pueden incluir desde preguntas } \\
\text { cerradas, que según las respuestas deseadas lo ubi- } \\
\text { quen en determinado cumplimiento de esas caracte- } \\
\text { rísticas o roles; o con preguntas abiertas que permi- } \\
\text { tan conocer directa e indirectamente si esa persona } \\
\text { reuniría esos requisitos } \\
\text { Sea siguiendo una u otra modalidad, se debe procu- } \\
\text { rar: } \\
\text { - Identificar los rasgos más significativos de su } \\
\text { personalidad } \\
\text { - Conocer las competencias que posee y los } \\
\text { puntos débiles para su desempeño profesio- } \\
\text { nal y como persona } \\
\text { - Los resultados de sus desempeños laborales } \\
\text { de los últimos años o meses (según corres- } \\
\text { ponda) } \\
\text { - Su trayectoria profesional y niveles de forma- } \\
\text { ción-capacitación alcanzados } \\
\text { - Opinión de sus jefes inmediatos y compañeros } \\
\text { (cuando corresponda y sea pertinente) } \\
\text { - Autoevaluación de si cumple con esas carac- } \\
\text { terísticas y puede realizar esas actuaciones } \\
\text { esperadas }\end{array}$ & $\begin{array}{l}\text { Beneficios: } \\
\text { - Obtener una valoración del rendimiento y la más } \\
\text { alta productividad } \\
\text { - Lograr mejorar su nivel organizacional y salarial } \\
\text { - Disfrutar más su trabajo } \\
\text { - Tener más conocimiento y visión de la organización } \\
\text { - Desarrollar mejor sus habilidades personales } \\
\text { - Aumentar su autoestima } \\
\text { - Adaptarse mejor a los cambios } \\
\text { - Incrementar sus conocimientos } \\
\text { - Ser más crítico y autocrítico } \\
\text { - Aprender a tomar conciencia de las percepciones } \\
\text { de otros } \\
\text { - Desarrollar habilidades de análisis y de toma de } \\
\text { decisiones } \\
\text { - Obtener incentivos (si la organización los ofrece co- } \\
\text { mo política) }\end{array}$ \\
\hline
\end{tabular}




\begin{tabular}{|c|c|}
\hline \multicolumn{2}{|c|}{ Coordinador } \\
\hline $\begin{array}{l}\text { Características } \\
\text { - Valora el aprendizaje organizacional } \\
\text { - Genera empoderación-empowerment en los otros } \\
\text { - Con grandes habilidades interpersonales } \\
\text { - Centrado: que tiene claro lo que quiere y espera } \\
\text { - Con sentido del humor y "buen temperamento" } \\
\text { - Seguro de sí mismo } \\
\text { - Humilde } \\
\text { - Autocrítico y receptivo a las críticas } \\
\text { - Organizado }\end{array}$ & $\begin{array}{l}\text { Actuaciones esperadas } \\
\text { - Guiar y mediar } \\
\text { - Elegir a los tutelados, tutores y a las parejas de tra- } \\
\text { bajo } \\
\text { - Ser capaz de comunicarse adecuadamente con } \\
\text { otros y divulgar efectivamente el programa } \\
\text { - Estar comprometido con la organización } \\
\text { - Ser diligente en la gestión de recursos y en las bue- } \\
\text { nas relaciones con la alta dirección } \\
\text { - Sistematizar y organizar las mejores prácticas y } \\
\text { lecciones aprendidas durante el desarrollo del pro- } \\
\text { grama }\end{array}$ \\
\hline $\begin{array}{l}\text { Criterios de selección } \\
\text { El coordinador es elegido normalmente porque cum- } \\
\text { ple una función en el organigrama de la organización } \\
\text { en el área de formación y de recursos humanos o, } \\
\text { en casos muy especiales, y en organizaciones muy } \\
\text { flexibles, horizontales, se designa a personas que tie- } \\
\text { nen grandes competencias en fomentar procesos de } \\
\text { aprendizaje en áreas-temáticas específicas, en este } \\
\text { caso sería en Alfabetización Informacional, además } \\
\text { de competencias gerenciales: planear, organizar, di- } \\
\text { rigir y evaluar }\end{array}$ & $\begin{array}{l}\text { Beneficios: } \\
\text { - Ser visto como un buen seleccionador de capital } \\
\text { humano: "cazatalentos" } \\
\text { - Trabajar con la alta dirección de la organización } \\
\text { - Ampliar su red de contactos (networking) } \\
\text { - Conocer más la organización } \\
\text { - Desarrollar habilidades de análisis y de toma de } \\
\text { decisiones } \\
\text { - Obtener incentivos (si la organización los ofrece co- } \\
\text { mo política) }\end{array}$ \\
\hline
\end{tabular}

Si concretamos estas características (cualidades), actuaciones y criterios para ser seleccionados en un programa de mentorización en ALFIN, implicarían adicional y específicamente:

- Mentores ALFIN

- Ser un profesional alfabetizado informacionalmente.

- Ser conocedor de los conceptos claves, las normas y modelos de ALFIN a nivel universitario.

- Haber sido formador en competencias informacionales.

- Ser muy didáctico. Ser un educador (Maestro).

- Tener experiencia y buen desempeño previo en actividades de formación y/o docentes.

- Tutelados ALFIN

- Tener conocimientos básicos del uso de las TIC, la gestión de información.

- Estar dispuesto al aprendizaje mediado por distintas tecnologías.

- Reconocer la importancia de la ALFIN para dar formación para toda la vida.

- Estar dispuesto en el futuro próximo a ser formador de ALFIN. 
- Coordinadores ALFIN

- Tener presente que la ALFIN es más que formar usuarios de modo tradicional.

- Darse cuenta de que ALFIN implica trabajo interdisciplinario.

- Saber que los mentores y tutelados son principalmente de la biblioteca pero procurarse la vinculación cada vez mayor de docentes e investigadores.

- Reconocer que la clave de ALFIN en la universidad es ser un programa transversal y curricular, que permite que la adquisición de competencias informacionales llegue a todos los estudiantes, en sus distintos años-grados y niveles de formación: pregrado y posgrado, y al personal académico-investigativo y administrativo.

\section{f. Formas propicias de trabajo}

Para desarrollar el trabajo de mentorización hay varias recomendaciones a partir de las lecciones aprendidas y mejores prácticas que estos mismos programas en diferentes tipos de organizaciones han generado:

- Iniciar el proceso poco a poco. Desarrollar un plan piloto, aprender, y luego hacerlo más grande, formal y con mayores alcances.

- Por cada mentor debe haber un número máximo de dos tutelados y su emparejamiento debe considerar similitudes o no, según lo que la organización y el programa mismo consideren más pertinente: edad, sexo, nivel de formación, cultura (en unos casos según el área, el que sean similares o no, es una ventaja). Para el caso de ALFIN se considera que la diferencia es un punto clave, por lo cual se deberá buscar que no haya mucha similitud, pero sí posibilidades de empatía.

- Cada participante debe conocer muy bien lo que su rol implica y los aspectos específicos que se esperan de él mismo (características y actuaciones esperadas), por lo que la formación inicial antes de comenzar el intercambio es fundamental, ya sea desarrollando conferencias, talleres, designando lecturas, etcétera.

- Un proceso de mentorización tiene diferentes fases, cada una de las cuales debe seguirse y tener objetivos específicos que finalmente faciliten la consecución de los objetivos y metas generales del programa:

- Inicio: El mentor y el tutelado deben consolidar la relación (generar empatía, conocerse y conocer sus expectativas, establecer 
y oficializar ante la organización los objetivos y metas a lograr en un tiempo específico y siendo lo más realistas posibles, planificar las sesiones y la forma de trabajo).

- Desarrollo: El mentor y el tutelado avanzarán en su relación y mantendrán un flujo constante y productivo de trabajo que poco a poco les rendirá beneficios a ambos y para la organización; a su vez, para lograr el mejoramiento continuo del programa. Serán capaces de avanzar y replantear rumbos de trabajo (flexibles), ser más críticos y autocríticos.

- Separación: Cuando se hayan conseguido los objetivos en el tiempo esperado, habrá llegado el momento de finalizar el programa de mentorización entre esta pareja (que luego con otras, cambiando roles, puede y debe seguir) por lo cual hay que sistematizar y evaluar los resultados, pero también el proceso, lo cual es clave para beneficio personal y organizacional para así hacer gestión del conocimiento del mismo e identificar las lecciones aprendidas y las mejores prácticas.

- Redefinición: El mentor y el tutelado deciden, ya por fuera del programa, como seguirá su relación de mutuo aprendizaje y que le aportarán a otros mentores y tutelados, al programa mismo de mentorización y a la organización, sin ser éste un compromiso formal. En esta fase la mentorización situacional o informal reaparece, con todos los beneficios que puede tener, pero conectada con el programa de mentorización formal, así aportándose, complementándose, una a la otra.

g. Recursos necesarios: materiales, tecnología, espacios físicos y tiempo

De no contar con los recursos necesarios, según el alcance que se le quiera dar al programa de mentorización, y en concreto a las relaciones entre los mentores y tutelados, es mejor no desarrollarlo o realizarlo con un corto alcance y un bajo número de mentores-tutelados.

Un programa de este tipo implica invertir en formación de los participantes y permitir la disponibilidad adecuada de materiales, tecnología (berramientas de comunicación vía Internet-Intranet y de gestión de conocimiento) y espacios físicos (sitio de reuniones que propicie el silencio para lograr un mejor intercambio y evitar las interrupciones) que permitan esta formación-autoformación, y los distintos momentos que un programa de este tipo implica, como se mencionó en el ítem anterior (Inicio, Desarrollo, Separación, Redefinición); por lo cual tener un presupuesto definido es una obligación. 
Adicionalmente un programa de mentorización implica disponibilidad de tiempo; es necesario que tanto para la formación como para el intercambio entre mentor y tutelado, y la mediación del coordinador, éstos dispongan de tiempo, aunque ello implique dejar por "un tiempo" sus labores habituales. Esto sólo se logra si en los directivos y entre mentores y tutores existe claridad sobre los beneficios personales y organizacionales que un programa de este tipo puede ofrecer. En el caso de ALFIN, si no se tiene clara su importancia para la universidad de hoy, no hay soporte para tal programa.

h. Evaluación continua y nuevos ciclos de mentorización

Llevando a cabo lo planificado, considerando los objetivos y metas, y realizando una retroalimentación constante (formativa y sumativa) con los participantes, se tienen las bases para alcanzar una buena evaluación de un programa de mentorización.

Es importante tener presente que un programa de mentorización implica un fuerte componente afectivo que permite el logro o no de lo esperado por el mentor y por el tutelado, por lo cual se debe tener muy presente tanto lo aprendido en términos de conocimientos y habilidades como lo vivido en términos de sentimientos, experiencias, aptitudes y actitudes, para así identificar bien las lecciones aprendidas, las mejores prácticas y lo que se debe considerar si esas personas quieren seguir en el programa (sea como mentores o tutelados).

Ante el desarrollo de las TIC, de nuevos modelos y normas de ALFIN, y de orientaciones didácticas-pedagógicas, es necesario enfatizar que además del programa de mentorización, tanto la universidad como la biblioteca deben proseguir, o desarrollar si aún no lo han hecho, un plan estructurado de capacitación que permita que los mentores experimentados, los potenciales mentores (los tutelados que tuvieron un buen desempeño) y los nuevos tutelados tengan la posibilidad de actualizarse.

Por tanto un programa de mentorización debe formar parte de una macroestrategia organizacional de gestión del conocimiento, donde la formación continua e integral del personal universitario en su conjunto sea un pilar importante.

\section{Conclusiones}

Los resultados en diferentes organizaciones, y en particular en las distintas bibliotecas universitarias reseñadas, muestran que éstos pueden ser muy buenos si se asume que toda organización moderna debe ser una organización que 
aprende, que gestiona su conocimiento, y que eso a corto y sobre todo mediano y largo plazo traerá grandes beneficios.

Concretamente para la ALFIN lograr que los bibliotecarios que tienen pocas competencias para ser formadores de otros en esta temática, las alcancen, es fundamental, pues difícilmente una universidad logrará estudiantes, profesores e investigadores competentes informacionalmente, si no tiene un buen programa de ALFIN que integre de manera nuclear y transversal la competencia informacional en todos los planes de estudio y en los programas de formación a lo largo de la vida.

En la medida en que se implemente un programa de mentorización en ALFIN y éste vaya presentando buenos resultados internos, crecerá y será referente para motivar a otras universidades, pues siempre hay que divulgar lo que estamos haciendo bien y el boca-oído es la mejor publicidad.

A su vez, en la realidad actual, donde se apuesta por el trabajo colaborativo y en red, trabajar mancomunadamente es esencial, por lo que la unión de las universidades y de sus bibliotecas para formar personas alfabetizadas informacionalmente es fundamental, más aún cuando en ciertos casos dada la situación particular de algunas bibliotecas universitarias (poco personal, personal más experimentado ya jubilado, etc.), algunos mentores, o los formadores en la capacitación inicial y continua de mentores-tutelados, podrían ser personas de otras instituciones que quieren compartir sus conocimientos sobre ALFIN.

Por tanto la mentorización es una opción estratégica que las bibliotecasCRAI a nivel mundial, y especialmente a nivel iberoamericano, pueden emplear para hacer que sus profesionales sean competentes y aprendan de las mejores prácticas tanto de docentes como de profesionales seniors, con el fin de construir una sociedad con mayor acceso a la información, que aprende en forma permanente y es crítica-autocrítica.

\section{BiBLIOGRAFÍA}

ACRL/ALA, (Association of College and Research Libraries/American Library Association), "Normas sobre aptitudes para el acceso y uso de la información en la educación superior", en Boletín de la Asociación Andaluza de Bibliotecarios0, Año 15, Núm. 60, 2000, disponible en: http://www.aab.es/pdfs/baab60/60a6.pdf (Consultado: 5-10-2001). 
ACRL/ALA, (Association of College and Research Libraries/American Library Association), "Características de los programas de alfabetización en información que sirven como ejemplo de las mejores prácticas", en Boletín de la Asociación Andaluza de Bibliotecarios, núm.70, pp. 67-72, 2003, disponible en: http://www.aab.es/ pdfs/baab70/70a4.PDF (Consultado: 22-04-2004).

Andey, M; Early, F; Foster, M., The mentors handbook, Londres: Herts Tec, 1999.

Arizona State University Libraries, K.1: Mentoring Program. Arizona Board of Regents. Disponible en: http://lib.asu.edu/ (consultado: 11-8-2009).

ASCMLA, Association of Canadian Map Libraries and Archives, Proposal for a Mentoring Program. 2002, disponible: www.ssc.uwo.ca/assoc/acml/carto2002/acmla_mentor.pdf (Consultado: 11-8-2009).

Ben, J; Brennand M. Australian Library and Information Association (ALIA), Why mentoring matters:

a personal journey that created professional success, 2008, disponible en: http://conferences.alia.org.au/ newlibrarian2008/Papers/Benn.doc (consultado: 4-9-2009).

Bey, T.M. y Holmes, C.T., Mentoring: Contemporary Principles and Issues, Boston, Association of Teachers Educators, 1992.

Black, W. K. y J. M. Leysen, "Fostering success: The socialization of entry-level librarians in ARL libraries", en Journal of Library Administration, 36(4), 3-27, 2002.

Bonnette, A.E., "Mentoring minority librarians up the career ladder, en Library Administration E Management, 18(3), 134-139, 2004.

Bullinngton, J.S. y Boylston, S.D., "Strengthening the Professional, Assuring our Future: ACRL's New Member Mentoring Program Pairs Library Leaders with New Professionals", en College E Research Libraries News, 62(4), 403-2, 2001.

ANZIIL (Australian and New Zealand Institute for Information Literacy), Bundy, A., Australian and New Zealand Information Literacy Framework: principles, standards and practice, 2nd. ed., Adelaide, 2004, disponible en: http://www.anziil.org/resources/Info \%20 lit\%202nd\% 20edition.pdf (consultado: 12-12-2004).

Cahill, L., y Blanchard, S.R., GWA Mentoring Handbook, Arizona State University. Graduate Women's Association, 2001, disponible en: http://www.asu.edu/clubs/gwa/GWAmentorhandbook.pdf (consultado: 11-8-2009).

Cornell University Library, CUL CPD's Mentorship Program, Committee on Professional Development, disponible en: www.library. cornell.edu/pdc/Mentor.html (consultado: 11-8-2009).

Cronan-Hillix, T. et al., "Students' views of mentors in psychology graduate training”, en Teaching of Psychology, 13, 3, pp., 123-7, 1986.

Culpepper, J. C., "Mentoring Academic Librarians: The Ultimate in Career Guidance", en College E Undergraduate Libraries, 7.2, 7181, 2000. 
Davidson, J.R., y Middleton, M.A. Networking, Networking, Networking: The Role of Professional Association Memberships in Mentoring and Retention of Science Librarians", en Science E Technology Libraries 27, núm. 1/2: 203-224, 2006.

Demirhan, A., "Developing Leadership Through Mentoring", en Florida Libraries, 48(2), 15-16, 2005.

Donnelly, K. M., "Librarian as teachers", en Dekker, M. (ed). Encyclopedia of Library and Information Science, New York: Taylor \& Francis, pp. 1545-1552, 2003.

Farmer, D.; Stockham, M. y Trussell, A., "Revitalizing a mentoring program for academic librarians", en College and research libraries, 70(1) pp. 8-24, 2009, disponible en: http://krex.k-state.edu/ dspace/handle/2097/1242 (consultado: 30-8-2009).

Flores, M.A., "Induction and mentoring. Policy and Practice", en Dangel, J.R. (ed.), Research on Teacher Induction. Teacher Education Yearbook, 2006, XIV, v. 37-66, Rowan and Littlefield Publishers, Toronto.

Florida Library Association, Leadership Development Mentoring Program, disponible en: http://hrapps.fsu.edu/mentor/ (consultado: 30-8-2009).

Garner, S.D., Final Report of High-Level Colloquium on Information Literacy and Lifelong Learning Alexandria, Washington DC IFLA, 2006, disponible en: http://archive.ifla.org/III/wsis/High-LevelColloquium.pdf (consultado: 04-01-2009).

Gibson, R., "Mentoring \& Libraries: A Bibliography", en Council On Library /Media Technicians, 2003, disponible en: http://colt.ucr. edu/bibmentoring.html (consultado: 30-8-2009).

Golian, L.M., "Fostering librarian leadership through mentoring", en Adult Learning 14, núm 1, 2003.

Golian, L.M. y Galbraith, M.W., Effective Mentoring Programs for Professional Library Development, Advances in Library Administration and Organization. Greenwich, CT: JAI Press, 2006.

Gómez, J. A. y Licea de Arenas, J., La alfabetización informacional: su reflejo en la formación de los bibliotecólogos y en los servicios de las bibliotecas de universidades públicas de México y España, VIII Encuentro EDIBCIC (Asociación de Educadores, Investigadores de Bibliotecología, Archivología, Ciencias de la Información y Documentación de Iberoamérica y el Caribe). México, D. F., Noviembre 12-14, 2008.

Guerra Pérez, Y. y Martí Lahera, Y. (2010), “La Alfabetización Informacional en la formación pre y posgradual de bibliotecarios. Breve mirada desde el análisis documental”, en Congreso INFO 2010-Cuba. Panel Alfabetización Informacional, disponible en: http://www.congreso-info.cu/UserFiles/File/Info\%202010/Trabajos/Guerra\%20P\% C3\%A9rez,\%20YosvanyINFO\%202010.doc (consultado: 10-05-2010) 
Haglund, L., "Mentoring as a tool for staff development", en Health Libraries Group. Health Information and Libraries Journal, 21, pp.61-65, 2004.

Hilbun, J. y Akin, L., "E-Mentoring for Librarians and Libraries”, en Texas Library Journal, 83(1), 29-32, 2007.

INFOLIT Global/IFLA-UNESCO, Information Literacy Repository (Products for users-Credit courses), disponible en: http://www.infolitglobal.net/directory/en/browse/category/products/credit_ courses (consultado: 19-06-2010).

Kuyper-Rushing, L., "A formal mentoring program in a university library: components of a successful experiment", en Acad Libr., Nov;27(6): pp. 440-6, 2001.

Lary, M., "Mentoring: A Gift for Professional Growth", en The Southeastern Libraries, 47, 23, 1998.

Lau, Jesús et al., Information Literacy: An international state-of-the art report, UNESCO-IFLA, 2007, disponible en: http://www.infolitglobal.info/es/ (consultado: 10-11-2008).

Licea de Arenas, J., "La alfabetización informacional en el entorno hispanoamericano", en Anales de documentación, Núm. 12, 2009, págs. 93-106, disponible en: http://dialnet.unirioja.es/servlet/ articulo ?codigo $=3000809 \&$ orden $=215926 \&$ info $=$ link $($ consul tado: 10-11-2008).

Library Leadership Network, Academic Library Mentoring Programs 2009, disponible en: http://ln.lyrasis.org/ node/22 (consultado: 30-08-2009).

Martorana, J. et al., "A focus on mentorship in career development", en Library Administration E Management 18, núm. 4, 2004.

Meloni, C., "Mentoring the Next Generation of Library Media Specialists”, en Library Media Connection, 24(4), 32-3, 2006.

Moir, E., Launching the next generation of theachers though quality induction, ERIC, 2003.

Mosley, P. A., "Mentoring Gen X managers: Tomorrow's library leadership is already here", en Library Administration E Management, 19(4), 185-92, 2005.

Mullen, C.A. y Lick, D.W., New directions in Mentoring. Creating an Culture of Synergy, London: Routledge Falmer, 1999.

Munde, G., "Beyond mentoring: toward the rejuvenation of academic libraries", en Journal of Academic Librarianship, Vol. 26 Núm.3, pp.171-5, 2000.

Nankivell, C. y Shoolbred, M., "Mentoring in Library and Information Services: A Literature Review and Report on Recent Research", en New Review of Academic Librarianship, 3: 91-144, 1997.

NJLA, "Recruiting and Mentoring a Diverse Workforce for New Jersey's Libraries", en New Jersey State Library and Urban Libraries Section of NJLA, panel moderator, Monroe Township Library, June 10, 2009, disponible en: http:/www.njstatelib.org/LDB/Events/ Past_Events.php (consultado: 30-8-2009). 
Penny, B., Providing feedback to employees. Armed forces comptroller, 42-44, Spring 2008.

Peer Systems Consulting Group Inc., A guide to the mentor program listings, 2009, disponible en: http://www.islandnet.com/ rcarr/ mentorprograms.html (consultado: 30-8-2009).

Pinto, M., Cordon, J.A. y Gomez, R., "Thirty years of Information Literacy (1977-2007): a terminological, conceptual and statistical analysis", en JOLIS, 2010, 42, Núm. 1, 3-19.

Pinto, M., Sales D. y Osorio, P., Biblioteca Universitaria, CRAI y alfabetización informacional, Ediciones TREA, 2008.

"El personal de la biblioteca universitaria y la alfabetización informacional: de la auto percepción a las realidades y retos formativos", en Revista Española de Documentación Cientifica, 32(1), enero-marzo, pp. 60-80, 2009.

Portmann C.A. y Jilius Roush A., "Assessing the effect of library instruction”, en The Journal of Academic Librarianship, 30 (6):461-5, 2004.

REBIUN, Plan Estratégico 2007-2010, disponible en: http://crue.org/rebiun (consultado: 25-07-2008).

, Competencias informáticas en informacionales en los estudios de grado, 2009, disponible en: http://www.rebiun.org/opencms/ opencms/handle404?exporturi=/export/docReb/documento_competencias_informaticas.pdf\&\%5d (consultado: 12-08-2009).

, Guia de buenas prácticas para el desarrollo de las competencias informacionales (adaptación de la guía de CAUL), 2008, disponible en: http:/www.rebiun.org/opencms/opencms/handle404?exporturi=/ export/ docReb/guia_buenas_practicas.doc\&\%5d (consultado: 1208-2009).

SCONUL, (Society of College, National and University Libraries. United Kingdom), Learning Outcomes and Information Literacy, London: Higher Education Academy, 2004, disponible: http://www. heacademy.ac.uk (consultado: 2-04-2008).

SELA, Southeastern Library Association Mentoring Program, disponible en: http://selaonline.org/membership/ mentoring.htm (consultado: 30-08-2009).

Shea, G.F., Mentoring: Helping Employees Reach their Full Potential, New York: Americam Management Association, 1994.

, Mentoring: A Practical Guide, Herts: Crisp publications, 1997.

Making the Most of Being Mentored: How to Grow a Mentoring Partnership, Herts: Crisp publications, 1999.

, Mentoring: how to develop successful mentor behaviors, Menlo Park: Crisp Learning, 2002.

, The mentoring organization, Menlo Park: Crisp Learning, 2003. 
Sit, A., Capitalizing on Knowledge: Mentorship among Teacher-Librarians in Hong Kong. Second Symposium on Field Experience. The Hong Kong Institute of Education Library, 2003, disponible en: http://www.ied.edu.hk/fesym/1A03-010\%20Full\%20paper.pdf (consultado: 2-08-2009).

Soler Angels, M.R., Mentoring: estrategia de desarrollo de recursos bumanos, Barcelona: Gestión 2000, 175, p. 2003.

Sowell, S.L., Education librarians, en Dekker, M. (editor), Encyclopedia of library and information science, New York: Taylor \& Francis, pp. 972- 976, 2003.

Special Libraries Association, The Mentorship Handbook: A Guide for SLA Chapters and Divisions to Establish Mentorship Programs, Baltimore: Special Libraries Association, 2001, disponible en: http:// www.sla.org/pdfs/mentorsh.pdf (consultado: 30-09-2009).

Studwell, W.E., Mentoring and the Future of Library and Information Science, Technicalities, 22(4), 3, 6, 2002.

UnESCO Education, The necessary utopia. Delors Report, París, 1996, disponible en: http://unesco.org/delors/utopia.htm (consultado: 18-06-2006).

University of Georgia Libraries, Mentor Program: Share What You Know, disponible en: http://www.libs.uga.edu/ (consultado: 1208-2009).

University of Maryland Libraries, Mentoring Program, http://www. lib.umd.edu/PASD/LPO/mentoring/ (consultado: 12-08-2009).

University of Southern California Libraries, Library Faculty Mentoring Program, 2001, http://www.usc.edu/libraries/ about/faculty/ Mentoring_Policy_2001.doc (consultado: 12-08-2009).

University of Utah. Librarian Mentoring Program at the University of Utah's, J. Willard Marriott Library, disponible en: http://www.lib. utah.edu/portal/site/marriottlibrary/ (consultado: 12-08-2009)

Uribe Tirado, A., "Los bibliotecólogos colombianos y la adquisición de competencias. Énfasis y tendencias en la actual formación en Tecnologías de la Información y la Comunicación”, en Revista Interamericana de Bibliotecología, vol. 30, núm. 1, pp. 135-166, 2006.

Diseño, implementación y evaluación de una propuesta formativa en alfabetización informacional mediante un ambiente virtual de aprendizaje a nivel universitario: caso Escuela Interamericana de Bibliotecología, Universidad de Antioquia, Tesis Maestría en Ingeniería, Línea Informática Educativa, Thesis, EAFIT University (Colombia). 2008, disponible en: http://eprints.rclis.org/15301/ (consultado: 2-10-2008). (2010a), "La Alfabetización Informacional en la Universidad. Descripción y Categorización según los Niveles de Integración de ALFIN. Caso Universidad de Antioquia”, en Revista Interamericana de Bibliotecología, vol. 33, núm. 1, pp. 31-83, 2010. 
(2010b), "Avances y perspectivas de ALFIN en Iberoamérica. Una mirada desde la publicación académico-científica y la web 1.0 y 2.0", en. Congreso INFO 2010, Cuba. Panel Alfabetización Informacional, disponible en: http://eprints.rclis.org/18597/ (consultado: 1-6-2010).

Vanzant, L., Achievement motivation, sex-role acceptance, and mentor relationships of professional females', unpublished doctoral dissertation, East Texas State University, Texas, 1980.

Wang, H., "Academic mentorship: An effective professional development strategy for medical reference librarians", en Medical Reference Services Quarterly, 20(2), 23-31, 2001.

Wasik J. A., Comprehensive VR Training Program, enLankes D, et. al., Virtual Reference Service: From Competencies to Assessment, New Jersey: Neal Schuman, pp.115 - 92, 2007.

Washington University Libraries, Library Mentor: Networking, Cooperation, Collaborations, Opportunity, 2007, disponible en: http:// wulibraries.typepad.com/mentor/ (consultado: 12-08-2009).

Webber, S. y Johnston, B., Working towards the information literate university, en Walton, G. and Pope, A. (Eds), Information literacy: recognising the need, Staffordshire University, Stoke-on-Trent: 17 May 2006. Oxford: Chandos, pp 47-58, 2006, disponible en: http://dis.shef.ac.uk/sheila/staffs-webber-johnston.pdf (consultado: 20-10-2009).

Webber, S., Information Literacy in Higher Education, en Stopar, K. and Rabzeljl. (Eds), Informacijska Pismenost med teorijo in prakso: vloga visokošolskih in specialnih knjižnic: Zbornik prispevkov, [Information Literacy between theory and practice: The role of academic and special libraries: Proceedings], Ljubljana: ZBDS. Pp. 9-20, 2006.

Wittkopf B., "Mentoring in academic libraries: LSU Libraries model", en LLA Bulletin Spring, 61(4):226-32, 1999.

Yale University Library, SCOPA Mentoring Program for the Yale University Library, 2007, disponible en: www.library.yale.edu/scopa/ mentoring/mentoring.html (consultado: 12-08-2009).

Zachary, L. J., The mentor's guide: facilitating effective learning relationships, San Francisco: Jossey-Bass Publishers, 2000. , Creating a mentoring culture: the organization's guide, John

Wiley \& Sons, 2005. 